\title{
Heckesche Systeme idealer Zahlen und Knesersche Körpererweiterungen
}

\author{
von \\ Toma Albu und Florin Nicolae (Bucureşti)
}

Einleitung. Eine klassische Konstruktion aus der algebraischen Zahlentheorie ist folgende: $\mathrm{Zu}$ jedem algebraischen Zahlkörper $K$ kann man ein sogenanntes System idealer Zahlen $S$ zuordnen, welches eine Untergruppe der multiplikativen Gruppe $\mathbb{C}^{*}$ der komplexen Zahlen ist derart, daß die Faktorgruppe $S / K^{*}$ in kanonischer Weise isomorph zu der Klassengruppe $C l_{K}$ von $K$ ist. Diese Konstruktion geht auf Hecke [5] zurück und hat folgende wichtige Eigenschaft, die auch bei dem Hilbertschen Klassenkörper zu $K$ vorkommt: Jedes Ideal von $K$ wird in $K(S)$ ein Hauptideal, wobei $K(S)$ den durch $K$ und $S$ erzeugten Unterkörper von $\mathbb{C}$ bezeichnet. Über den Grad $[K(S): K]$ behauptet Hecke, daß $[K(S): K]=\left|C l_{K}\right|$ sei; wir konnten aber keinen Beweis dieser Behauptung in der Literatur finden. Der Zweck unserer Arbeit ist einen sehr kurzen und einfachen Beweis der Gleichheit $[K(S): K]=\left|C l_{K}\right|$ zu geben, mittels eines schönen Satzes von Kneser [7]. Diese Gleichheit gilt allgemeiner für den Quotientenkörper eines Dedekindschen Ringes.

1. Terminologie und Grundbegriffe. In diesem Abschnitt bezeichnet $K$ einen beliebigen Körper, $\bar{K}$ seinen algebraischen Abschluß und für jede natürliche Zahl $n \geq 1$ ist $\zeta_{n}$ eine primitive $n$-te Einheitswurzel, d.h. eine Erzeugende der zyklischen Gruppe $\left\{z \in \bar{K} \mid z^{n}=1\right\}$.

Ist $G$ eine beliebige Gruppe, so schreiben wir $H \leq G$, wenn $H$ eine Untergruppe von $G$ ist. Ist nun $M$ eine Teilmenge von $G$, so bezeichnen wir mit $\langle M\rangle$ die durch $M$ erzeugte Untergruppe von $G$. $\operatorname{Ord}(g)$ bezeichnet die Ordnung eines Elementes $g \in G$ und $\operatorname{Exp}(G)$ den Exponenten von $G$. Für jeden Körper $L$ bezeichnen wir mit $L^{*}$ die multiplikative Gruppe aller von Null verschiedenen Elemente aus $L$. Schließlich, werden wir die Kardinalzahl einer Menge $X$ durch $|X|$ bezeichnen.

Der folgende Satz von Kneser ist für unsere Arbeit grundlegend: 
SATz 1.1 ([7; Satz]). Sei $L / K$ eine endliche separable Körpererweiterung und $G$ eine Gruppe mit $K^{*} \leq G \leq L^{*}$ und endlicher Faktorgruppe $G / K^{*}$, so daß $L=K(G)$ gilt. Dann und nur dann ist $\left|G / K^{*}\right|=[L: K]$, wenn für ungerade Primzahlen p jede zu $G$ gehörige p-te Einheitswurzel $\zeta_{p}$ schon in $K$ liegt, und wenn $\zeta_{4}$ in $K$ liegt, falls $1+\zeta_{4}$ in $G$ ist.

Der obige Satz führte uns zu der folgenden Definition:

Definition 1.2 ([1; Definition 2.2]). Sei $L / K$ eine Körpererweiterung und $G$ eine Gruppe. Die Erweiterung $L / K$ heißt eine $G$-Knesersche Erweiterung, falls $L / K$ eine endliche Erweiterung ist, mit $K^{*} \leq G \leq L^{*}$, $L=K(G)$ und $\left|G / K^{*}\right|=[L: K]$. Die Erweiterung $L / K$ heißt eine Knesersche Erweiterung, falls $L / K$ eine $G$-Knesersche Erweiterung für eine bestimmte Gruppe $G$ ist.

Behauptung 1.3. Sei $L / K$ eine separable $G$-Knesersche Erweiterung und $H$ eine Gruppe mit $K^{*} \leq H \leq G$. Dann ist die Erweiterung $K(H) / K$ eine $H$-Knesersche Erweiterung.

B eweis. Wir wollen beweisen, daß $[K(H): K]=\left|H / K^{*}\right|$ ist. Dafür wenden wir den Satz 1.1 von Kneser an. Sei $p$ eine ungerade Primzahl mit $\zeta_{p}$ in $H$. Dann gehört $\zeta_{p}$ auch zu $G$. Da $K \subseteq K(G)$ eine $G$-Knesersche Erweiterung ist, folgt aus 1.1, daß $\zeta_{p}$ in $K$ liegt. Nehmen wir an, daß $1+\zeta_{4}$ in $H$ liegt. Dann liegt $1+\zeta_{4}$ auch in $G$, und aus 1.1 folgt es wieder, daß $\zeta_{4}$ in $K$ liegt. Also sind die Bedingungen des Kneserschen Satzes 1.1 für die Erweiterung $K(H) / H$ erfüllt.

2. Das Hauptergebnis. Sei $K$ ein algebraischer Zahlkörper, $\mathcal{O}_{K}$ der Ring aller ganzen Zahlen von $K, I_{K}$ die Gruppe der gebrochenen Ideale von $K, H_{K}$ die Gruppe der Hauptideale von $K, C l_{K}=I_{K} / H_{K}$ die Klassengruppe von $K, h=\left|C l_{K}\right|$ die Anzahl der Idealklassen. Sei $\mathcal{C}_{1}, \ldots, \mathcal{C}_{s}, s \geq 1$ eine Basis der Abelschen Gruppe $C l_{K}$ : Jede Klasse $\mathcal{C} \in C l_{K}$ besitzt eine eindeutige Darstellung

$$
\mathcal{C}=\mathcal{C}_{1}^{r_{1}} \ldots \mathcal{C}_{s}^{r_{s}},
$$

wobei $0 \leq r_{k}<h_{k}, h_{k}$ die Ordnung der Klasse $\mathcal{C}_{k}, k=1, \ldots, s, h=h_{1} \ldots h_{s}$ ist. Sei, für jedes $k=1, \ldots, s, I_{k}$ ein ganzes Ideal der Klasse $\mathcal{C}_{k}$. Dann besitzt jedes gebrochene Ideal $I \in I_{K}$ eine eindeutige Darstellung

$$
I=(a) I_{1}^{r_{1}} \ldots I_{s}^{r_{s}},
$$

$a \in K^{*}, 0 \leq r_{k}<h_{k}, k=1, \ldots, s$, wobei die Exponenten $r_{k}$ eindeutig bestimmt sind. Wir haben mit $(a)$ das gebrochene Ideal $a \mathcal{O}_{K}$ bezeichnet.

Wegen $\mathcal{C}_{k}^{h_{k}}=1$ gilt es $I_{k}^{h_{k}}=\left(c_{k}\right) \in H_{K}$ mit Zahlen $c_{k} \neq 0$ aus $K$, $k=1, \ldots, s$, die nur bis auf willkürliche Einheitsfaktoren aus $K$ festliegen. 
Wir denken uns die Zahlen $c_{k}$ fest gewählt und bilden den Zahlkörper

$$
L:=K\left(\gamma_{1}, \ldots, \gamma_{s}\right) \text {, }
$$

wobei $\gamma_{k}$ eine Wurzel des Polynoms $X^{h_{k}}-c_{k}$ über $K$, also $\gamma_{k}^{h_{k}}=c_{k}$ sei. Die Zuordnung

$$
\text { (a) } I_{1}^{r_{1}} \ldots I_{s}^{r_{s}} \mapsto \gamma_{1}^{r_{1}} \widehat{\ldots} \gamma_{s}^{r_{s}},
$$

$a \in K^{*}, 0 \leq r_{k}<h_{k}, k=1, \ldots, s$, induziert einen Gruppenhomomorphismus

$$
\psi_{K}: I_{K} / H_{K} \rightarrow K^{*}\left\langle\gamma_{1}, \ldots, \gamma_{s}\right\rangle / K^{*},
$$

wobei $\widehat{\gamma}$ die Klasse des Elementes $\gamma$ von $K^{*}\left\langle\gamma_{1}, \ldots, \gamma_{s}\right\rangle$ in der Faktorgruppe $K^{*}\left\langle\gamma_{1}, \ldots, \gamma_{s}\right\rangle / K^{*}$ ist. (Siehe auch [8; S. 506].)

Offensichtlich, ist $\psi_{K}$ surjektiv. Wir zeigen nun, daß tatsächlich $\psi_{K}$ einen Gruppenisomorphismus ist. Dafür genügt es zu beweisen, daß $r_{1}=\ldots=$ $r_{s}=0$ ist, falls $c:=\gamma_{1}^{r_{1}} \ldots \gamma_{s}^{r_{s}} \in K^{*}$ ist, $0 \leq r_{k}<h_{k}, k=1, \ldots, s$. In der Tat, betrachten wir das ganze Ideal $I=I_{1}^{r_{1}} \ldots I_{s}^{r_{s}}$. Wegen $I_{k}^{h_{k}}=\left(\gamma_{k}\right)^{h_{k}}=$ $\left(c_{k}\right)$, gilt $\left(I_{k} \mathcal{O}_{L}\right)^{h_{k}}=\left(\gamma_{k} \mathcal{O}_{L}\right)^{h_{k}}$ für jedes $k=1, \ldots, s$, und es folgt daraus $I_{k} \mathcal{O}_{L}=\gamma_{k} \mathcal{O}_{L}, k=1, \ldots, s$. Folglich ist

$$
I \mathcal{O}_{L}=\left(\gamma_{1}^{r_{1}} \ldots \gamma_{s}^{r_{s}}\right) \mathcal{O}_{L}=c \mathcal{O}_{L},
$$

und so ist

$$
I=I \mathcal{O}_{L} \cap \mathcal{O}_{K}=c \mathcal{O}_{L} \cap \mathcal{O}_{K}=c \mathcal{O}_{K} .
$$

Die Klasse $\mathcal{C}$ von $I$ in $C l_{K}$ ist also die Hauptklasse. Da $\mathcal{C}=\mathcal{C}_{1}^{r_{1}} \ldots \mathcal{C}_{s}^{r_{s}}$ ist, folgt es $r_{1}=\ldots=r_{s}=0$, was zu zeigen war.

Aus (**) folgt also unmitellbar folgende Gleichheit:

$$
\left|K^{*}\left\langle\gamma_{1}, \ldots, \gamma_{s}\right\rangle / K^{*}\right|=\left|I_{K} / H_{K}\right|=h .
$$

Aus dem obigen Beweis folgen:

(1) Die Zahlen von $(a) I_{1}^{r_{1}} \ldots I_{s}^{r_{s}}$ mit $a \in K^{*}$ sind mit denjenigen Zahlen von $K$ identisch, welche durch $a \gamma_{1}^{r_{1}} \ldots \gamma_{s}^{r_{s}}$ teilbar sind (d.h., es ist $a \gamma_{1}^{r_{1}} \ldots$ $\left.\ldots \gamma_{s}^{r_{s}} \mathcal{O}_{L} \cap K=(a) I_{1}^{r_{1}} \ldots I_{s}^{r_{s}}\right)$;

(2) Das Ideal $(a) I_{1}^{r_{1}} \ldots I_{s}^{r_{s}}$ geht in das Hauptideal $\left(a \gamma_{1}^{r_{1}} \ldots \gamma_{s}^{r_{s}}\right)$ des Körpers $K\left(a \gamma_{1}^{r_{1}} \ldots \gamma_{s}^{r_{s}}\right)$ über.

Hecke nennt die Gruppe $K^{*}\left\langle\gamma_{1}, \ldots, \gamma_{s}\right\rangle$ ein System idealer Zahlen zu $K$ und behauptet, daß der Grad $[L: K]=h$ sei, ohne einen Beweis zu geben [6; S. 122].

Bezüglich derselben Problematik sagt Hasse: “... auf die Frage nach der Irreduzibilität der Polynome $X^{h_{k}}-c_{k}$ über $K$ wollen wir hier nicht eingehen" [4; S. 544] und Ribenboim gibt nur die Ungleichung $[L: K] \leq h$ $[9 ;$ S. 124] an. 
Mann kann sowohl die Behauptung von Hecke als auch die Irreduzibilität der Polynome $X^{h_{k}}-c_{k}$ über $K$ sehr einfach mittels des Satzes 1.1 von Kneser beweisen:

SATz 2.1. Mit den obigen Bezeichnungen ist $[L: K]=h$.

Wir brauchen den folgenden Hilfssatz, der zuerst von Hecke in [5; S. 18] zum Ausdruck gebracht worden ist.

Hilfssatz 2.2. Sei $\varepsilon$ eine Einheit von $L$, die in $K^{*}\left\langle\gamma_{1}, \ldots, \gamma_{s}\right\rangle$ liegt. Dann gehört $\varepsilon$ zu $K$.

B ew eis. Nehmen wir an, daß $\varepsilon=a \gamma_{1}^{r_{1}} \ldots \gamma_{s}^{r_{s}}$ ist, $a \in K^{*}, 0 \leq r_{k}<h_{k}$, $k=1, \ldots, s$. Wegen $I_{k}^{h_{k}}=\left(\gamma_{k}\right)^{h_{k}}=\left(c_{k}\right)$ für jedes $k=1, \ldots, s$, gilt $\left(I_{k} \mathcal{O}_{L}\right)^{h_{k}}=\left(\gamma_{k} \mathcal{O}_{L}\right)^{h_{k}}$, und es folgt daraus $I_{k} \mathcal{O}_{L}=\gamma_{k} \mathcal{O}_{L}, k=1, \ldots, s$. Also, hat das Ideal $I=(a) I_{1}^{r_{1}} \ldots I_{s}^{r_{s}}$ von $K$ die Eigenschaft

$$
I \mathcal{O}_{L}=\left(a \gamma_{1}^{r_{1}} \ldots \gamma_{s}^{r_{s}}\right) \mathcal{O}_{L}=(\varepsilon) \mathcal{O}_{L}=\mathcal{O}_{L}
$$

Wir erhalten

$$
I=I \mathcal{O}_{L} \cap K=\mathcal{O}_{L} \cap K=\mathcal{O}_{K}=(1) .
$$

Die Klasse $\mathcal{C}$ von $I$ in $C l_{K}$ ist also die Hauptklasse. Da $\mathcal{C}=\mathcal{C}_{1}^{r_{1}} \ldots \mathcal{C}_{s}^{r_{s}}$ ist, folgt $r_{1}=\ldots=r_{s}=0$, und $\varepsilon=a$ ist ein Element von $K$.

Beweis von Satz 2.1. Wir wollen beweisen, daß

$$
\left[K\left(\gamma_{1}, \ldots, \gamma_{s}\right): K\right]=\left|K^{*}\left\langle\gamma_{1}, \ldots, \gamma_{s}\right\rangle / K^{*}\right|=h
$$

ist. Da $K\left(\gamma_{1}, \ldots, \gamma_{s}\right)=K\left(K^{*}\left\langle\gamma_{1}, \ldots, \gamma_{s}\right\rangle\right)$ ist, reicht es, die Bedingungen des Satzes 1.1 von Kneser zu prüfen. Sei $p$ eine ungerade Primzahl mit $\zeta_{p}$ in $K^{*}\left\langle\gamma_{1}, \ldots, \gamma_{s}\right\rangle$. Aus 2.2 folgt es, daß $\zeta_{p}$ in $K$ liegt. Nehmen wir jetzt an, daß $1+\zeta_{4}$ in $K^{*}\left\langle\gamma_{1}, \ldots, \gamma_{s}\right\rangle$ liegt. Dann liegt auch $\left(1+\zeta_{4}\right)^{2}=2 \zeta_{4}$ in $K^{*}\left\langle\gamma_{1}, \ldots, \gamma_{s}\right\rangle$, so liegt $\zeta_{4}$ in $K^{*}\left\langle\gamma_{1}, \ldots, \gamma_{s}\right\rangle$. Nach 2.2 liegt $\zeta_{4}$ in $K$. Die Kneserschen Bedingungen sind also erfüllt. Aus 1.1 folgt es

$$
\left[K\left(\gamma_{1}, \ldots, \gamma_{s}\right): K\right]=\left|K^{*}\left\langle\gamma_{1}, \ldots, \gamma_{s}\right\rangle / K^{*}\right|=h .
$$

Folgerung 2.3. Sei $\mathcal{C}$ eine Idealklasse von $K$ und $\widehat{\gamma}$ das zugeordnete Element in die Gruppe $K^{*}\left\langle\gamma_{1}, \ldots, \gamma_{s}\right\rangle / K^{*}$ durch den Isomorphismus $\psi_{K}$. Sei $m=\operatorname{Ord}(\mathcal{C})$ in $C l_{K}$. Dann ist $[K(\gamma): K]=m$ und jedes Ideal von $\mathcal{C}$ wird ein Hauptideal in $K(\gamma)$.

Beweis. In der Tat, ist die Gruppe $K^{*}\langle\gamma\rangle$ eine Untergruppe von $K^{*}\left\langle\gamma_{1}, \ldots, \gamma_{s}\right\rangle$, also erfüllt sie (nach 1.3) auch die Kneserschen Bedingungen, so ist

$$
[K(\gamma): K]=\left|K^{*}\langle\gamma\rangle / K^{*}\right|=\operatorname{Ord}(\widehat{\gamma})=\operatorname{Ord}(\mathcal{C})=m .
$$

B emerkung 2.4. Der Körper $L=K\left(\gamma_{1}, \ldots, \gamma_{s}\right)$ besitzt zwei Eigenschaften, die auch bei dem Hilbertschen Klassenkörper $K_{0}$ zu $K$ vorkommen: Es ist $[L: K]=h$, und jedes Ideal von $K$ wird in $L$ ein Hauptideal. Im 
allgemeinen sind $L$ und $K_{0}$ verschieden, weil $L / K$ im allgemeinen keine Galoissche Erweiterung ist. Die Folgerung 2.3 zeigt, daß jede Idealklasse $\mathcal{C} \in C l_{K}$ von der Ordnung $m$ in die Hauptklasse eines Zwischenkörpers von $L / K$ vom Grade $m$ über $K$ übergeht. Die Frage, ob $K_{0}$ dieselbe Eigenschaft besitzt, ist von Artin und Furtwängler negativ beantwortet worden. (Siehe [3; S. 173-174].)

SATz 2.5. Sei $I \in I_{K}$ ein Ideal von $K$ und $m=\operatorname{Ord}(\mathcal{C})$, wobei $\mathcal{C}$ die Klasse von $I$ in $\mathrm{Cl}_{K}$ ist. Sei $c \in K^{*}$ mit $I^{m}=(c)$. Dann ist das Polynom $X^{m}-c$ irreduzibel in $K[X]$.

B ew eis. Sei $\gamma$ eine Wurzel von $X^{m}-c$. Im Körper $K(\gamma)$ ist $I \mathcal{O}_{K(\gamma)}=$ $\gamma \mathcal{O}_{K(\gamma)}$. Sei $\varepsilon$ eine Einheit von $\mathcal{O}_{K(\gamma)}$ mit $\varepsilon \in K^{*}\langle\gamma\rangle, \varepsilon=a \gamma^{r}, a \in K^{*}, 0 \leq$ $r<m$. Man erhält $I^{r}=\left(I \mathcal{O}_{K(\gamma)}\right)^{r} \cap K=(\gamma)^{r} \mathcal{O}_{K(\gamma)} \cap K=\left(\varepsilon a^{-1}\right) \mathcal{O}_{K(\gamma)} \cap$ $K=\left(a^{-1}\right) \mathcal{O}_{K(\gamma)} \cap K=\left(a^{-1}\right) \mathcal{O}_{K}$. Es folgt $m \mid r$, also ist $r=0$, und $\varepsilon=a$ gehört zu $K^{*}$. Wir verfahren nun wie im Beweis von 2.1, um zu zeigen, daß $K(\gamma) / K$ eine $K^{*}\langle\gamma\rangle$-Knesersche Erweiterung ist. Also ist $[K(\gamma): K]=$ $\left|K^{*}\langle\gamma\rangle / K^{*}\right|=\operatorname{Ord}(\widehat{\gamma})$. Sei $n=\operatorname{Ord}(\widehat{\gamma})$. Es folgt $n \mid m$. Im Körper $K$ haben wir $I^{n}=\left(\gamma^{n}\right)$, also ist $I^{n}$ ein Hauptideal. Es folgt $m \mid n$, so ist $m=n$. Also ist $[K(\gamma): K]=m$, und somit ist der Polynom $X^{m}-c$ irreduzibel in $K[X]$.

Folgerung 2.6. Mit den obigen Bezeichnungen gelten die folgenden Aussagen:

(1) Für jedes $k=1, \ldots, s$ sind die Polynome $X^{h_{k}}-c_{k}$ irreduzibel über $K$.

(2) Die Körper $K\left(\gamma_{1}\right), \ldots, K\left(\gamma_{s}\right)$ sind linear disjunkt über $K$.

(3) Es gibt einen kanonischen Gruppenisomorphismus

$$
K^{*}\left\langle\gamma_{1}, \ldots, \gamma_{s}\right\rangle / K^{*} \simeq \prod_{1 \leq i \leq s}\left(K^{*}\left\langle\gamma_{i}\right\rangle / K^{*}\right)
$$

Beweis. (1) folgt unmittelbar aus 2.5.

(2) Wegen (1), gilt $\left[K\left(\gamma_{j}\right): K\right]=h_{j}$ für $j=1, \ldots, s$, und somit ist

$$
\left[K\left(\gamma_{1}, \ldots, \gamma_{s}\right): K\right]=h=h_{1} \ldots h_{s}=\left[K\left(\gamma_{1}\right): K\right] \ldots\left[K\left(\gamma_{s}\right): K\right] .
$$

Das zeigt, daß die Körper $K\left(\gamma_{1}\right), \ldots, K\left(\gamma_{s}\right)$ linear disjunkt über $K$ sind.

(3) ist klar, wegen des Isomorphismus

$$
\psi_{K}: I_{K} / H_{K} \rightarrow K^{*}\left\langle\gamma_{1}, \ldots, \gamma_{s}\right\rangle / K^{*} .
$$

B e merkung 2.7. Wenn $\gamma_{k}^{\prime}$ eine andere Wurzel des Polynoms $X^{h_{k}}-c_{k}$, $k=1, \ldots, s$, ist, kann man zeigen, daß die Körper $K\left(\gamma_{1}, \ldots, \gamma_{s}\right)$ und $K\left(\gamma_{1}^{\prime}, \ldots, \gamma_{s}^{\prime}\right)$ isomorph über $K$ sind, mittels des Isomorphismus $\gamma_{k} \mapsto \gamma_{k}^{\prime}$, $k=1, \ldots, s$.

In [1] haben die Authoren die folgende Frage gestellt: Wie könnte man die Zwischenkörper einer $G$-Kneserschen Erweiterung bestimmen? Für eine 
$G$-Knesersche Erweiterung $L=K(G)$ kommen die folgenden kanonischen Abbildungen $\alpha$ und $\beta$ vor:

$$
\begin{aligned}
\alpha: \mathcal{L} \rightarrow \mathcal{G}, & \alpha(E)=E \cap G, \\
\beta: \mathcal{G} \rightarrow \mathcal{L}, & \beta(H)=K(H),
\end{aligned}
$$

wobei

$$
\begin{aligned}
& \mathcal{G}=\left\{H \mid K^{*} \leq H \leq G\right\}, \\
& \mathcal{L}=\{E \mid K \subseteq E, E \text { Unterkörper von } L\},
\end{aligned}
$$

ist. Der günstigste Fall ist jener, wenn $\alpha$ und $\beta$ inverse Bijektionen sind, und in [1] ist dafür eine notwendige und hinreichende Bedingung formuliert worden.

SATZ 2.8 ([1; Theorem 3.7]). Sei $L / K$ eine separable Erweiterung mit $L=K(G), K^{*} \leq G \leq L^{*}$ und endlicher Faktorgruppe $G / K^{*}$ vom Exponenten $n=\operatorname{Exp}\left(G / K^{*}\right)$. Mit den obigen Bezeichnungen sind die folgenden Behauptungen äquivalent:

(1) $L / K$ ist eine $G$-Knesersche Erweiterung und $\alpha$ und $\beta$ sind inverse Isomorphismen von Verbänden;

(2) $L / K$ ist eine $n$-reine Erweiterung, d.h. für jede Primzahl $p$ oder $p=4$ mit $p \mid n$ liegt $\zeta_{p}$ in $K$, falls $\zeta_{p}$ in $L$ ist.

Im Falle einer Galoisschen Erweiterung $L / K$ gibt es einen Antiisomorphismus zwischen dem Verband $\mathcal{L}=\{E \mid K \subseteq E, E$ Unterkörper von $L\}$ und dem Verband aller Untergruppen einer kanonischen Gruppe, nämlich, die Galoissche Gruppe $\operatorname{Gal}(L / K)$, die zu $L / K$ zugeordnet ist. Die Erweiterungen aus 2.8 haben eine duale Eigenschaft: Es gibt einen Isomorphismus zwischen $\mathcal{L}$ und dem Verband aller Untergruppen der Gruppe $G / K^{*}$. Wegen dieser dualen Eigenschaft, nennen wir die Erweiterungen wie in 2.8 G-kogaloissche Erweiterungen.

Es entsteht nun die natürliche Frage, ob die oben betrachtete Erweiterung $(*) K\left(\gamma_{1}, \ldots, \gamma_{s}\right) / K$ eine $K^{*}\left\langle\gamma_{1}, \ldots, \gamma_{s}\right\rangle$-kogaloissche Erweiterung ist. Die Antwort ist negativ.

Betrachten wir zum Beispiel den Körper $K=\mathbb{Q}(\sqrt{-87})$, für den $h=$ 6 ist. (Siehe die Tabellen aus [2].) $\mathrm{Da}-87 \equiv 1(\bmod 4)$ ist, ist $\mathcal{O}_{K}=$ $\mathbb{Z}\left[\frac{1+\sqrt{-87}}{2}\right]$. Die Primidealzerlegung von 3 in $\mathcal{O}_{K}$ ist $3 \mathcal{O}_{K}=(3, \sqrt{-87})^{2}$. Das Primideal $I=(3, \sqrt{-87})$ ist kein Hauptideal. In der Tat, nehmen wir an, daß $I=(\delta)$ ist, mit einer Zahl

$$
\delta=a+b \frac{1+\sqrt{-87}}{2}
$$

aus $\mathcal{O}_{K}$. Wir bezeichnen mit $N=N_{K / \mathbb{Q}}$ die Normabbildung der Erweiterung 
$K / \mathbb{Q}$. Es folgt $N(\delta) \mid N(3)=9$ und $N(\delta) \mid N(\sqrt{-87})=87$, so ist

$$
N(\delta)=\left(a+\frac{b}{2}\right)^{2}+87 \frac{b^{2}}{4}=a^{2}+a b+22 b^{2}=3 .
$$

Diese Gleichung ist unlösbar im Ring der ganzen rationalen Zahlen: Für $a b \geq 0$ ist das offenbar; wenn $a b<0$ ist, folgt es $a^{2}+b^{2}>-a b$ und $21 b^{2} \geq$ $21>3$, und wir erhalten $a^{2}+22 b^{2}>3-a b$, und so ist $a^{2}+a b+22 b^{2}>3$.

Die Klasse $\mathcal{C}_{1}$ von $I$ in $C l_{K}$ hat also die Ordnung 2 , und es ist $I^{2}=$ $(3)=(-3)$. Wir wählen $\gamma_{1}=\sqrt{-3}$ als ideale Zahl für $I$. Die Gruppe $C l_{K}$ ist zyklisch von der Ordnung 6 , so gibt es eine Klasse $\mathcal{C}_{2}$ von der Ordnung 3 , und $\left\{\mathcal{C}_{1}, \mathcal{C}_{2}\right\}$ ist eine Basis von $C l_{K}$. Sei $\gamma_{2}$ eine ideale Zahl für ein ganzes Ideal von $\mathcal{C}_{2}$. Die Gruppe $K^{*}\left\langle\sqrt{-3}, \gamma_{2}\right\rangle$ ist ein System idealer Zahlen zu $K$. Nach 2.1 ist die Erweiterung $L / K$ mit $L=K\left(\sqrt{-3}, \gamma_{2}\right)$ eine $K^{*}\left\langle\sqrt{-3}, \gamma_{2}\right\rangle$ Knesersche Erweiterung. Man hat $\operatorname{Exp}\left(K^{*}\left\langle\sqrt{-3}, \gamma_{2}\right\rangle / K^{*}\right)=6,3 \mid 6$ und $\zeta_{3}=(1+\sqrt{-3}) / 2$ gehört zu $L$, so ist die Erweiterung $L / K$ keine 6 -reine Erweiterung. Aus 2.8 folgt es, daß $L / K$ keine $K^{*}\left\langle\sqrt{-3}, \gamma_{2}\right\rangle$-kogaloissche Erweiterung ist.

Jedoch, wenn der Grundkörper $K$ eine reele Einbettung besitzt, können wir die idealen Zahlen $\gamma_{1}, \ldots, \gamma_{s}$ derart bilden, daß die Erweiterung $L / K$ mit $L=K\left(\gamma_{1}, \ldots, \gamma_{s}\right)$ eine $K^{*}\left\langle\gamma_{1}, \ldots, \gamma_{s}\right\rangle$-kogaloissche Erweiterung ist. Ohne Beschränkung der Allgemeinheit können wir annehmen, daß $K \subseteq \mathbb{R}$ ist. In der obigen Konstruktion der idealen Zahlen können wir $c_{1}>0, \ldots, c_{s}>0$ wählen. Für $\gamma_{k}$ wählen wir die positive reele Wurzel des Polynoms $X^{h_{k}}-c_{k}$, $1 \leq k \leq s$. Dann ist $L=K\left(\gamma_{1}, \ldots, \gamma_{s}\right)$ ein reeler Körper, der außer 1 und -1 keine Einheitswurzeln mehr enthält, so ist $L / K$ in trivialer Weise eine $n$-reine Erweiterung für irgendwelche natürliche Zahl $n$. Nach 2.8 ist $L / K$ eine $K^{*}\left\langle\gamma_{1}, \ldots, \gamma_{s}\right\rangle$-kogaloissche Erweiterung.

Gleichfalls, wenn $\zeta_{h}$ in $K$ liegt, dann ist die Erweiterung $L / K$ mit $L=$ $K\left(\gamma_{1}, \ldots, \gamma_{s}\right)$ eine $K^{*}\left\langle\gamma_{1}, \ldots, \gamma_{s}\right\rangle$-kogaloissche Erweiterung. (Siehe [1; Theorem 5.2].)

Es sei endlich bemerkt, daß alle oben festgestellten Ergebnisse für Dedekindsche Ringe verallgemeinert werden können:

Sei $A$ ein Dedekindscher Ring mit endlicher Idealklassengruppe $C l_{A}$ von der Ordnung $h$ und Quotientenkörper $K$. Nehmen wir an, daß die Charakteristik von $K$ verschieden von 2 und relativ prim zu $h$ ist. In diesem Falle können wir die am Anfang des Abschnitts 2 durchgeführte Konstruktion mutatis mutandis wiederholen, und damit können wir ein System idealer Elementen $K^{*}\left\langle\gamma_{1}, \ldots, \gamma_{s}\right\rangle z u K$ bilden. Wir erhalten:

SATz 2.9. Sei A ein Dedekindscher Ring mit endlicher Idealklassengruppe von der Ordnung $h$ und Quotientenkörper $K$. Wir nehmen an, daß die Charakteristik von $K$ verschieden von 2 und relativ prim $z u h$ ist. Dann gelten für $A$ die Behauptungen aus 2.1, 2.3, 2.5 und 2.6. 


\section{Literatur}

[1] T. Albu and F. Nicolae, Kneser field extensions with cogalois correspondence, J. Number Theory 52 (1995), 299-318.

[2] S. I. Borevič und I. R. Š afarevič, Zahlentheorie, Birkhäuser, Basel, 1966.

[3] H. Hasse, Bericht über neuere Untersuchungen und Probleme aus der Theorie der algebraischen Zahlkörper, Teil II: Reziprozitätsgesetz, Physica-Verlag, Würzburg, 1965.

[4] - Zahlentheorie, Akademie-Verlag, Berlin, 1963.

[5] E. Hecke, Eine neue Art von Zetafunktionen und ihre Beziehungen zur Verteilung der Primzahlen (Zweite Mitteilung), Math. Z. 4 (1920), 11-51.

[6] - Vorlesungen über die Theorie der algebraischen Zahlen, Chelsea, New York, 1948.

[7] M. Kneser, Lineare Abhängigkeit von Wurzeln, Acta Arith. 26 (1975), 307-308.

[8] J. Neukirch, Algebraische Zahlentheorie, Springer, Berlin, 1992.

[9] P. Ribenboim, Algebraic Numbers, Wiley, New York, 1972.

Toma Albu

FACULTATEA DE MATEMATICĂ

UNIVERSITATEA BUCUREŞTI

STR. ACADEMIEI 14

RO-70109 BUCUREŞTI 1, ROMANIA

E-mail: TALBU@IMAR.RO
Florin Nicolae

INSTITUTUL DE MATEMATICĂ AL ACADEMIEI ROMÂNE

P.O. BOX 1-764

RO-70700 BUCUREŞTI 1, ROMANIA

E-mail: FNICOLAE@IMAR.RO 